\title{
Connectivity of frontoparietal regions reveals executive attention and consciousness interactions
}

\author{
Martín-Signes, Mar $^{1}$, Paz-Alonso, Pedro M. ${ }^{2}$, Chica, Ana B. ${ }^{1}$ \\ ${ }^{1}$ Department of Experimental Psychology, and Mind, Brain, and Behavior Research \\ Center (CIMCYC), University of Granada, Spain. \\ ${ }^{2}$ BCBL, Basque Center on Cognition, Brain, and Language, Donostia, Spain.
}

Correspondence should be addressed to Mar Martín-Signes. Centro de Investigación Mente, Cerebro y Comportamiento (CIMCYC). Universidad de Granada. Campus de Cartuja S/N. CP 18071. Granada, Spain. Phone number: 00349582478 79. Contact email: msignes@ugr.es.

Number of words for Abstract: 173; Introduction: 1047; Methods: 2750; Results: 1064; Discussion: 1557; Number of Figures: 6; Number of tables: 3.

Running title: Neural mechanisms of executive attention and consciousness.

The authors declare no conflict of interest. 


\begin{abstract}
The executive control network is involved in the voluntary control of novel and complex situations. Solving conflict situations or detecting errors have demonstrated to impair conscious perception of near-threshold stimuli. The aim of this study was to explore the neural mechanism underlying executive control and its interaction with conscious perception using functional magnetic resonance imaging (fMRI) and diffusion-weighted imaging (DWI). To this end, we used a dual-task paradigm involving Stroop and conscious detection tasks with near-threshold stimuli. A set of prefrontal and frontoparietal regions were more strongly engaged for incongruent than congruent trials while a distributed set of frontoparietal regions showed stronger activation for consciously than non-consciously perceived trials. Functional connectivity analysis revealed an interaction between executive control and conscious perception in frontal and parietal nodes. The microstructural properties of the middle branch of the superior longitudinal fasciculus (SLF) were associated with neural measures of the interaction between executive control and consciousness. These results demonstrate that conscious perception and executive control share neural resources in frontoparietal networks, as proposed by some influential models.
\end{abstract}

Keywords: conscious perception, executive control, functional magnetic resonance imaging (fMRI), Superior Longitudinal Fascicle (SLF). 


\section{INTRODUCTION}

In our daily life, we are able to perform a wide set of tasks without deliberate attention or awareness. However, a different sort of actions appear to require attentional resources (Norman and Shallice 1986). Executive control operates when our acting schemas are ineffective, impossible, or insufficient to lead with a specific situation, such as those that involve planning, novelty, error or conflict detection/resolution (Posner and Digirolamo 1998; Diamond 2013). The executive control network is one of the three main attentional networks proposed by Petersen and Posner, together with alerting and spatial orienting (Posner and Petersen 1990; Petersen and Posner 2012).

Attentional processes can either boost conscious perception when aligned to the target or the relevant dimensions, or impair consciousness when attention is away, as demonstrated in many previous observations (Shapiro et al. 1997; Simons and Levin 1997; Solomon 2004). Based on this evidence, some theories propose that attention is a gateway for conscious perception (Posner 1994; Dehaene and Naccache 2001; Dehaene et al. 2006; Chica and Bartolomeo 2012). According to these proposals, research examining the effects of alerting and spatial orienting attentional networks over conscious perception has revealed that these attentional subsystems can interact with consciousness differently (Botta, Lupiáñez, \& Chica, 2014; Chica, Botta, Lupiáñez, \& Bartolomeo, 2012; Chica, Lasaponara, Lupiáñez, Doricchi, \& Bartolomeo, 2010; Koch \& Tsuchiya, 2007; Kusnir, Chica, Mitsumasu, \& Bartolomeo, 2011; Petersen, Petersen, Bundesen, Vangkilde, \& Habekost, 2017; Wyart \& Tallon-Baudry, 2008). Nevertheless, the role of the executive network on conscious processing remains largely under-explored. 
Recently, Colás and collaborators used a dual-task paradigm combining a detection task of near-threshold stimuli with a Stroop task. Results revealed a modulation of the decision criteria to detect the near-threshold stimuli when they were presented concurrently with the Stroop task, which was reflected in the modulation of the N2 potential, and associated with the activation of the anterior cingulate cortex (ACC) (Colás et al. 2017, 2018). Furthemore, in dualtask situations, in which executive control is also required, conscious perception can be delayed or impaired (Pashler 1994; Meyer and Kieras 1997; Shapiro et al. 1997). These results suggest that executive control elicited by conflict situations influences perception or decision stages of conscious processing through the involvement of frontal regions on both conflict monitoring/resolution (Szameitat et al. 2002; Egner and Hirsch 2005; Fan et al. 2005) and conscious perception (Rees et al. 2002; Rounis et al. 2010; Lau and Rosenthal 2011).

Early studies on the neural underpinnings of executive control associated Stroop effects with the functioning of frontal regions, such as the dorsolateral prefrontal cortex (dIPFC) and the ACC (Pardo et al. 1990; Macdonald et al. 2000; Milham et al. 2001; Miller and Cohen 2001; Egner and Hirsch 2005; Fan et al. 2005; Nee et al. 2007). According to the conflict monitoring theory, these two frontal structures have complementary roles: while the ACC evaluates and monitors the presence of conflict, the dIPFC implements cognitive control (Macdonald et al. 2000; Botvinick et al. 2001; Nee et al. 2007). In addition to the ACC and dIPFC, there is agreement on the implication of a wider set of regions in executive control processes, including the dorsal pre-motor cortex, supplementary motor area (SMA), inferior frontal 
junction, anterior insula, and posterior parietal cortex (Cole and Schneider 2007; Cocchi et al. 2013). Dosenbach and collaborators proposed that this set of regions is organized into two distinct networks: the frontoparietal and the cingulo-opercular networks. The former network would exert a rapid-active control using feedback information to affect processing of the succeeding item, while the latter might constitute a set-maintenance system that integrates the received information to exert proactive control (Dosenbach et al. 2008). Other models propose a central role to the cingulo-opercular network in switching from the default mode network to the frontoparietal control network (Bressler and Menon 2010).

Anatomically, parietal and frontal cortical regions are structurally connected by the Superior Longitudinal Fasciculus (SLF), a fiber tract organized in three parallel longitudinal branches: dorsal (i.e. SLF I), middle (i.e. SLF II), and ventral (i.e. SLF III) (Thiebaut de Schotten et al. 2011; Rojkova et al. 2016). Previous evidence has linked white matter microstructure of the SLF II and III with exogenous and endogenous spatial orienting in healthy populations (Thiebaut de Schotten et al. 2011; Carretié et al. 2012) and in patients with signs of spatial neglect (Doricchi et al. 2008; Ciaraffa et al. 2013; Thiebaut De Schotten et al. 2014; Vallar et al. 2014; Bourgeois et al. 2015). Sustained attention has been linked with the microstructural properties of the right SLF in typically developing children (Klarborg et al. 2013) and in individuals with attention-deficit/hyperactivity disorder (Konrad et al. 2010; Chiang et al. 2015; Wolfers et al. 2015). Recently, the neural interaction between conscious perception and different attentional subsystems (phasic alerting and exogenous orienting) has been 
related to the microstructure of the SLF III (Martín-Signes et al. 2017; Chica et al. 2018). However, to our knowledge, there is no evidence so far relating SLF microstructure with executive attention in the healthy population.

In the present study, we investigated the neural bases of the interaction between executive control and consciousness using functional magnetic resonance imaging (fMRI) and diffusion-weighted imaging (DWI) tractography. In the scanner, participants performed a Stroop task concurrently with a conscious detection task of near-threshold Gabor stimuli (see also Colás, Capilla, \& Chica, 2018; Colás et al., 2017). Behaviorally, we expected to observe impaired Gabor detection for incongruent trials as compared to congruent trials (Colás et al. 2017). At the neural level, we expected to observe a distributed frontoparietal network more strongly engaged for consciously seen as compared to unseen Gabors. In addition, frontal regions, such as the ACC, the dIPFC, or the insula, should be more engaged during incongruent trials, in which executive control strategies are required, as compared to congruent trials. If executive control modulates conscious perception, then brain activations associated with executive control should be related to subsequent conscious reports. We expected to find neural interactions between conscious perception and executive control in the activation of the above-mentioned frontoparietal regions or in the functional connectivity among them. Finally, using DWI tractography, we explored to what extent the microstructural properties of the different branches of the SLF were associated with behavioral and functional correlates of executive control and its interaction with consciousness. 


\section{METHODS}

\section{Participants}

A sample of 20 right-handed volunteers [11 females, mean age 25.70 years, standard deviation $(S D)=3.34$ ] took part in the study. Participants were inexperienced with the task and reported to have a normal or correctedto-normal vision, normal color-discrimination, and Spanish as their native language. Participants had no neurological or psychiatric conditions and followed all the safety requirements to undergo MRI studies. They signed an informed consent form to participate in the experiment, and received a monetary compensation for their time and effort (10€/hour). The study was reviewed and approved by the Ethics Committee of the University of Granada, and was carried out in compliance with the recommendations of the Helsinki Declaration.

\section{Apparatus and stimuli}

E-prime software was used to control the presentation of stimuli, timing operations, and behavioral data collection (Schneider et al. 2002). Images were presented in a screen (NNL, 32", $1024 \times 768,60 \mathrm{~Hz}$ ) located at the back of the scanner and viewed with a mirror mounted on the head coil. Two markers $\left(3^{\circ}\right.$ height $\times 5.3^{\circ}$ width $)$ and a central fixation point $\left(0.4^{\circ} \times 0.4^{\circ}\right)$ were displayed against a grey background at the beginning of the trial. Each marker consisted of a black square outline, placed $4.5^{\circ}$ to the left and right of the fixation point. Spanish words for blue ("azul", $0.4^{\circ}$ height $\times 1.6^{\circ}$ width), green ("verde", $0.4^{\circ}$ height $\times 2^{\circ}$ width), and yellow ("amarillo", $0.4^{\circ}$ height $\times 3^{\circ}$ width) colors were presented $0.6^{\circ}$ above the fixation point. Words were displayed 
either in blue, green, or yellow ink (Figure 1). Trials were sorted as congruent when the word meaning and the ink color matched, and as incongruent when the word meaning and the ink color were different. The target was a Gabor stimulus that could appear inside the lateral boxes. Matlab 8.1 (http://www.mathworks.com) was used to create 100 Gabor stimuli (4 cycles/deg. spatial frequency, $1.8^{\circ}$ in diameter, SD of $0.1^{\circ}$ ), with a maximum and minimum Michelson contrast of 0.92 and 0.02 , respectively. Target contrast was manipulated before the experimental task in order to adjust the percentage of consciously-perceived targets to $\sim 50 \%$ (see Procedure section).

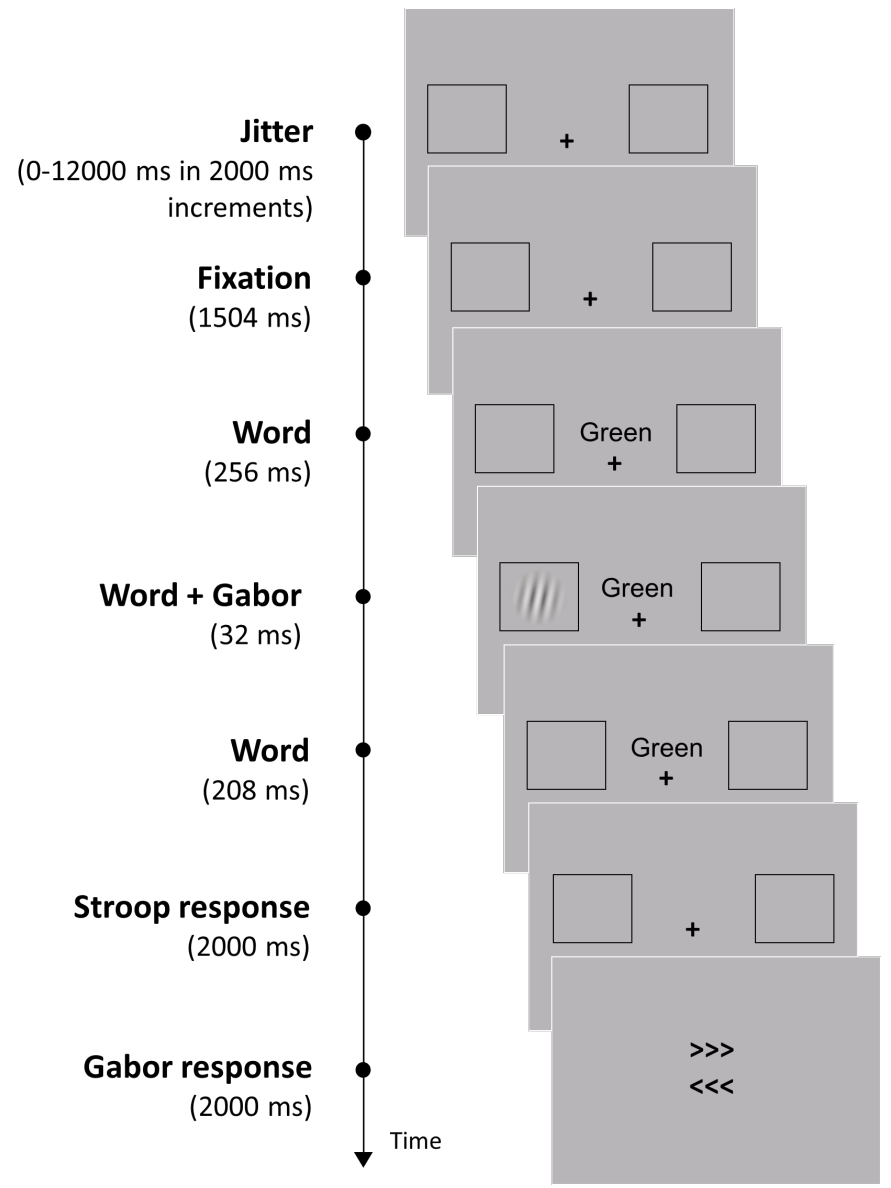

Figure 1. Sequence and timing of events in a trial of the experimental task. Words were displayed either in blue, green, or yellow ink. The example shows a trial with the Gabor present. 


\section{Procedure}

The timing and sequence of the events presented in a trial are depicted in Figure 1. In each trial, participants were presented with a color word and a Gabor stimulus (although $25 \%$ of the trials were catch trials, in which the Gabor was not presented). Participants were required to perform two consecutive tasks. First, they had to discriminate the word's ink color as fast and accurately as possible. Participants responded to this task with the index, middle, and ring finger of their right hand (color-key mapping counterbalanced across participants) using a 6-horizontally-aligned-button fiber-optic box. Second, participants had to report if they consciously detected the appearance of the Gabor. They were asked to respond as accurately as possible and only when they were confident about their perception. The response was given by choosing one of the two arrow-like stimuli (>>> or $\ll<$ ) pointing to the two possible locations of target appearance (right or left box). The arrows were presented one above the other, with their position randomized in each trial. Participants were required to indicate the location of the target, with the left hand, using a 6-aligned-button fiber-optic box positioned vertically. They used the ring finger to press an upper key (corresponding to the upper arrow), and the middle finger to press a lower key (corresponding to the bottom arrow). This was done to avoid response preparation before the subjective response was executed. If they had not perceived the Gabor, participants were asked to use the index finger to press a third key. Participants were explicitly instructed to fixate the central plus sign throughout all the experiment. 
In the scanner, but before the experimental trials, Gabor contrast was titrated for each participant to ensure that the percentage of seen targets would be $\sim 50 \%$ in both sessions. Titration began with a supra-threshold stimulus (Michelson contrast $=0.184$ ), whose contrast was manipulated in successive blocks depending on the mean percentage of seen targets after every 8 trials. After each block, if participants reported seeing $63 \%$ or more targets, Gabors at the immediately following lower contrast level (Michelson contrast minus 0.009 ) were used during the next block of trials; besides, if the percentage of seen targets was equal or lower than $38 \%$, the next block of trials used Gabors at the immediately following higher contrast level (Michelson contrast plus 0.009). The titration procedure stopped when target contrast yielded a percentage of seen targets ranging between $\geq 38 \%$ and $\leq 63 \%$ for two consecutive blocks of trials.

The experiment consisted of two sessions with 5 functional scans each. Each functional scan lasted for approximately 8 minutes. Across both sessions, participants encountered a total of 600 trials. Congruent and incongruent trials were presented in a pseudorandomized order during scanning. Incongruent trials accounted for $20 \%$ of the experimental trials. The Gabor was present on $75 \%$ of the trials, and absent on the remaining $25 \%$ of the trials (catch trials). The jitter fixation and the order of trial types within each scan were determined with an optimal sequencing program (i.e., Optseq2), designed to maximize the efficiency of recovery of the Blood-Oxygen-Level Dependent $\quad$ (BOLD) response 1999; http://surfer.nmr.mgh.harvard.edu/optseq/). The jitter fixation periods were 
interleaved with the experimental trials as determined by the optimization program.

\section{MRI data acquisition}

Functional and structural images were collected on a 3-T Siemens Trio MRI scanner at the Mind, Brain, and Behavior Research Center (CIMCYC, University of Granada), using a 32-channel whole-head coil. Functional images were acquired using a gradient-echo echo-planar pulse sequence [Repetition Time $(T R)=2000 \mathrm{~ms}$, Echo Time $(T E)=25$ ms, 35 interleaved 3.4-mm cubic axial slides, no inter-slice gap, flip angle $=75^{\circ}$, Field of View $(F O V)=220 \mathrm{~mm}, 345$ volumes per run]. Prior to each functional scan, several volumes were discarded to allow for saturation of the signal. High-resolution T1-weighted anatomical images $\left(\mathrm{TR}=2530 \mathrm{~ms}, \mathrm{TE}=3.5 \mathrm{~ms}\right.$, flip angle $=7^{\circ}$, slice thickness $=1 \mathrm{~mm}, \mathrm{FOV}=256 \mathrm{~mm}$ ) were also collected.

Additionally, a total of 70 near-axial slices were acquired using a sequence fully optimized for tractography of DWI providing isotropic 2-mm resolution and coverage of the whole head with a posterior-anterior phase of acquisition (TR $=8400 \mathrm{~ms}$ and $\mathrm{TE}=88 \mathrm{~ms})$. At each slice location, 6 images were acquired with no diffusion gradient applied and 60 diffusion-weighted images in which gradient directions were uniformly distributed in space. The diffusion weighting was equal to a b-value of $1500 \mathrm{sec} \mathrm{mm}^{2}$.

\section{fMRI data analysis}

SPM8 (Welcome Department of Cognitive Neurology, London) was used to conduct standard preprocessing routines and analyses. Images were corrected for differences in timing of slice acquisition and were realigned to 
the first volume by means of rigid-body transformation. Then, functional images were spatially smoothed using a 4-mm full width at half-maximum (FWHM) isotropic Gaussian kernel. Next, motion parameters obtained from realignment were used to inform a volume repair procedure (ArtRepair; Stanford Psychiatric Neuroimaging Laboratory) that identified bad volumes on the basis of within-scan movement and signal fluctuations, and then corrected bad signal values via interpolation. A volume-by-volume correction with a 1.5 $\mathrm{mm}$ threshold was applied, which did not correct more than $12 \%$ of the total volumes in any participant. After volume repair, structural and functional volumes were corregistered and spatially normalized to T1 and echo-planar imaging templates, respectively. The normalization algorithm used a 12parameter affine transformation together with a non-linear transformation involving cosine basis functions. During normalization, the volumes were sampled to 3-mm cubic voxels. Templates were based on the MNI305 stereotaxic space. Then, functional volumes were spatially smoothed with a 7mm FWHM isotropic Gaussian kernel. Finally, a $128 \mathrm{sec}$ high-pass filter was used to eliminate contamination from slow drift of signals.

Statistical analyses were performed on individual participants' data using the general linear model (GLM). fMRI time series data were modeled by a series of events convolved with a canonical hemodynamic response function (HRF). Three phases of each fMRI trial were modeled separately (stimuli presentation, Stroop response, and Gabor response). The model was created to examine the neural changes restricted to the stimuli-presentation period and was used in whole-brain contrast, regions-of-interest (ROIs), and functional connectivity analysis. Congruent and incongruent trials were sorted 
as seen or unseen in agreement to participants' responses. Accordingly, this model included regressors for the conditions: congruent seen, congruent unseen, incongruent seen, and incongruent unseen. Catch trials, errors, Stroop response and Gabor response periods, were modeled separately and excluded from the main analysis. All coordinates along the manuscript are reported in Montreal Neurological Institute (MNI) atlas space (Cocosco et al. 1997).

Contrast images, computed on a participant-by-participant basis were submitted to group analysis. At the group level, whole-brain contrasts between conditions were computed by performing one-sample t-tests on these images, treating participants as a random effect. Whole-brain maps involving all participants were thresholded at $q<0.05$ [false discovery rate (FDR) correction voxel wise] for target present versus jitter fixation (i.e., null events) contrast. ROI analyses were performed with the MARSBAR toolbox to use with SPM8 (Brett et al. 2002). ROls consisted of significantly active voxels identified from the Target present $>$ Null whole-brain functional contrast $(q<$ 0.05, voxel-wise FDR corrected) across all participants within a specific MARSBAR anatomical ROls. A set of ROls (the center of mass and the volume in $\mathrm{mm}^{3}$ are indicated between parentheses) were built, including frontal: left $\operatorname{ACC}\left(-8,21,29 ; 416 \mathrm{~mm}^{3}\right)$, right $\operatorname{ACC}\left(10,24,26 ; 552 \mathrm{~mm}^{3}\right)$, left frontal eye field (FEF; -24, 10, 49; $560 \mathrm{~mm}^{3}$ ), right FEF $\left(36,-1,52 ; 552 \mathrm{~mm}^{3}\right)$, left inferior frontal gyrus (IFG; -39, 24, 19; $5088 \mathrm{~mm}^{3}$ ), right IFG $(41,24,18$; $\left.4512 \mathrm{~mm}^{3}\right)$, left insula $\left(-32,19,4 ; 4608 \mathrm{~mm}^{3}\right)$, right insula $(36,21,1 ; 3416$ $\mathrm{mm}^{3}$ ), left middle frontal gyrus (MFG; $\left.-33,32,25,1976 \mathrm{~mm}^{3}\right)$, right MFG (37, 34,$\left.23 ; 1416 \mathrm{~mm}^{3}\right)$, left SMA $\left(-6,3,54 ; 7048 \mathrm{~mm}^{3}\right)$, right SMA $(8,9,54 ; 4128$ 
$\mathrm{mm}^{3}$ ); and parietal regions: left inferior parietal lobe (IPL; -36, -46, 45; 6592 $\mathrm{mm}^{3}$ ), right IPL $\left(32,-51,48 ; 1240 \mathrm{~mm}^{3}\right)$, left superior parietal lobe (SPL; -23, 61,$\left.49 ; 4080 \mathrm{~mm}^{3}\right)$, and right $\operatorname{SPL}\left(26,-59,53 ; 1512 \mathrm{~mm}^{3}\right)$. For each ROI, we performed a repeated-measures analysis of variance (ANOVA) on the parameter estimates values, with the factors congruency and awareness.

Finally, we assessed functional connectivity via the beta series correlation method (Rissman, Gazzaley, \& D’Esposito, 2004) implemented in SPM8 with custom Matlab scripts. The canonical HRF in SPM was fit to each occurrence of each condition and the resulting parameter estimates (beta values) were sorted according to the study conditions of interest (congruency: incongruent/congruent, and awareness: seen/unseen) to produce a conditionspecific beta series for each voxel. Two different functional connectivity analyses were performed: (1) pairwise functional connectivity between the regions showing the main effect of congruency (i.e., bilateral IFG, left MFG, bilateral SMA, left FEF, left IPL, and bilateral SPL) in the ROls analyses. Although the congruency effect was marginal for the left ACC ( $F=3.86$, MSE $\left.=8.84, p=0.06, \eta_{p}^{2}=0.17\right)$, we added this region to the pairwise functional connectivity analysis given its relevance in executive control (Macdonald, Cohen, Stenger, \& Carter, 2010; Milham et al., 2001; Nee, Wager, \& Jonides, 2007; Pardo, Pardo, Janer, \& Raichle, 1990); and (2) whole-brain functional connectivity with the left ACC as the seed region.

First, using pairwise functional connectivity analyses we calculated beta-series correlation values for each pair of ROls, condition, and participant. As indicated, these correlation values were obtained including all the trials in our fMRI experimental design assigned to each of the conditions. To identify 
significant coupling strength between ROls in each condition of interest these beta-series correlation values were averaged and two-tailed tests were used to determine the statistical significance of these $r$ values correcting for multiple comparisons ( $q<0.05, F D R)$. Then, to examine interactions in pairwise functional connectivity between these ROls, due to the fact that correlation coefficients are inherently restricted to range from -1 to +1 , an archyperbolic tangent transform was applied to these beta-series correlation values to make its null hypothesis sampling distribution approach that of the normal distribution (Fisher 1921). These Fisher's z normally distributed values were then submitted to repeated-measures ANOVAs with the factors Congruency and Awareness. Statistically significant interactions were followed by Tukey post-hoc analyses to examine the effects determining these interactions.

Second, for whole-brain functional connectivity analysis, the beta series associated with the left ACC were correlated with voxels across the entire brain to produce beta-correlation images. Contrasts between beta-correlation images were also subjected to an archyperbolic tangent transform to allow for statistical inference based on temporally coupled fluctuations with this region. Congruent seen $>$ Null, Congruent unseen $>$ Null, Incongruent seen $>$ Null, and Incongruent unseen $>$ Null t-tests were performed on the resulting subject contrast images to produce group correlation contrast maps with a threshold of $q<0.05$ (voxel-wise FDR corrected).

Statistical analyses were performed with STATISTICA 8.0 (StatSoft, Inc., 2007) and JASP 0.9.0.1 (JASP Team, 2018) softwares. Data and codes related to this paper can be accessed on 
https://www.bcbl.eu/Datasharing/CerebCor2018-MartinSignes-PazAlonso-

Chica/.

\section{DWI tractography analysis}

In each slice, diffusion-weighted data were simultaneously registered and corrected for subject motion and geometrical distortion adjusting the gradient accordingly (ExploreDTI, Leemans et al. 2009).

Individual dissections of the tracts were carried out with the software TrackVis (Wang et al. 2007). The three branches of the SLF (on the left and the right hemisphere) were isolated using a multiple region of interest approach. Three frontal ROIs around the white matter of the superior, middle and inferior frontal gyri and a ROI around the white matter of the parietal lobe were delineated. A no-part ROI in the temporal white matter was used to exclude streamlines of the arcuate fasciculus projecting to the temporal lobe (Thiebaut de Schotten et al. 2011; Rojkova et al. 2016). A new index, employed as a surrogate for tract microstructural organization (i.e., mean Hindrance Modulated Orientational Anisotropy, HMOA; Dell'Acqua et al. 2013), was extracted from each dissected tract on the left and right hemispheres.

Subsequently, we conducted Pearson correlations analysis using Z scores. Given the low number of subjects for these correlational analyses $(N=$ 19), a Bayesian approach was taken in addition to the Null Hypothesis Significance Testing in order to examine the probability of the data given the alternative hypothesis $(\mathrm{H} 1)$ relative to the null hypothesis $(\mathrm{H} 0)$ (i.e., Bayes factor $(\mathrm{BF})_{10}<1 / 3$ evidence favor $\mathrm{H} 0 ; \mathrm{BF}_{10}>3$ evidence favor $\mathrm{H} 1 ; 1 / 3<\mathrm{BF}_{10}$ $<3$ indicates data insensitivity) (Dienes and Mclatchie 2018). The mean 
HMOA of the left and right SLF I, II and III was correlated with the congruency effect over the percentage of seen targets (\% of seen targets for congruent minus incongruent condition). We also calculated an interaction index for the functional connectivity data of the three pairs of regions showing the interaction effect (left ACC and left IFG, left FEF and left IPL, and right SMA and right SPL). This index was calculated over the beta values, according to the following formula: beta values for seen minus unseen trials for the congruent minus incongruent condition.

\section{Behavioral data analysis}

Stroop RTs shorter than 150 ms were considered outliers and were eliminated from the analysis $(0.59 \%$ of the trials, $S D=0.54)$. Anticipatory responses were also excluded $(0.21 \%$ of the trials, $S D=0.05)$. False alarms (FA; trials in which participants consciously reported a Gabor that was not presented) accounted for only $4.9 \%(S D=6.86)$ of the catch trials and were excluded from the analyses. Errors localizing a consciously seen Gabor $(3.2 \%$ of the trials, $S D=0.17$ ) were also excluded.

We analyzed mean accuracy and reaction times (RTs) for the Stroop task and the percentage of seen targets for the Gabor detection task by means of repeated-measured ANOVAs with the within-participant factor of Congruency (congruent/incongruent). We also analyzed participants' responses to the Gabor detection task by using the signal detection theory (SDT, Abdi 2007). We computed a nonparametric index of perceptual sensitivity $\left(A^{\prime}\right)$ and response criterion ( $\left.\beta^{\prime \prime}\right)$ to detect the Gabor by using the following equations: 


$$
A^{\prime}=0.5+\frac{(\text { Hits }-F A s) *(1+\text { Hits }-F A s)}{4 * H i t s *(1-F A s)} ; \beta^{\prime \prime}=\frac{H i t s *(1-H i t s)-F A s *(1-F A s)}{H i t s *(1-H i t s)+F A s *(1-F A s)}
$$

$A^{\prime}$ values usually range between 0.5 (the signal cannot be distinguished from noise) to 1 (perfect performance). For $\beta "$, values close to 1 indicate a conservative criterion while values close to -1 indicate a nonconservative criterion (Stanislaw and Todorov 1999). These indexes were also submitted to two repeated-measures ANOVAs with the within-participant factor of Congruency.

\section{RESULTS}

\section{Behavioral results}

For the Stroop task, we observed the expected Congruency effect. Mean accuracy was significantly higher for congruent compared to incongruent trials, $F(1,19)=19.15, \mathrm{MSE}=0.0001, \mathrm{p}<0.001, \eta_{\mathrm{p}}^{2}=0.50$, and RTs were also significantly shorter for congruent compared to incongruent trials, $F(1,19)=54.20, M S E=1941, p<0.001, \eta_{p}^{2}=0.74$.

For the Gabor detection task, the percentage of seen targets was marginally larger for the congruent compared to the incongruent condition, $F(1,19)=4.10$, MSE $=0.001, p=0.057, \eta_{p}^{2}=0.18$. When the analysis was repeated including the RT for incongruent trials minus RT for congruent trials as a covariate, the main effect of congruency on the percentage of seen targets reached statistical significance, $F(1,19)=4.46$, MSE $=0.001, p=$ $0.049, \eta_{p}^{2}=0.20$, demonstrating that the congruency effect on the percentage of seen targets was larger for those participants with larger congruency effects on RTs. 
SDT analyses revealed that Congruency did not influence either perceptual sensitivity $\left(A^{\prime}\right)$ or response criterion $\left(\beta^{\prime \prime}\right)$ to detect the Gabor (all ps $\geq 0.22$ ). However, Congruency modulated the proportion of seen Gabors (see above) but not the proportion of FA (repeated-measured ANOVA comparing false alarms on congruent and incongruent conditions, $F(1,19)=0.13, \mathrm{MSE}=$ 0.009, $p=0.723, \eta 2 p=0.007)$. Table 1 shows the mean and SD of the analyzed measures for congruent and incongruent trials.

Table 1. Behavioral data for the Gabor detection task and for the Stroop task for congruent and incongruent trials.

\begin{tabular}{|c|c|c|c|c|}
\hline & \multicolumn{2}{|c|}{ Congruent } & \multicolumn{2}{|c|}{ Incongruent } \\
\hline & Mean & SD & Mean & SD \\
\hline Seen trials (\%) & $\underline{54}$ & $\underline{16}$ & $\underline{52}$ & 17 \\
\hline FA (\%) & $\underline{4.98}$ & $\underline{6.61}$ & 4.80 & $\underline{7.26}$ \\
\hline$\underline{A^{\prime}}$ & $\underline{0.85}$ & $\underline{0.06}$ & $\underline{0.85}$ & $\underline{0.07}$ \\
\hline$\underline{\beta}$ & $\underline{0.73}$ & $\underline{0.29}$ & $\underline{0.76}$ & $\underline{0.31}$ \\
\hline Stroop accuracy (\%) & $\underline{98}$ & $\underline{4.12}$ & $\underline{97}$ & $\underline{4.07}$ \\
\hline Stroop RT (ms) & $\underline{1091}$ & $\underline{169}$ & 1194 & $\underline{135}$ \\
\hline
\end{tabular}

\section{fMRI results}

The whole-brain contrast Target present $>$ Null revealed the regions that demonstrated larger BOLD responses when the Gabor was presented as compared to fixation. Increased activations were found bilaterally in the cingulate cortex, FEFs, inferior and middle frontal gyri, superior and inferior parietal lobes, SMA, insula, inferior and middle temporal gyri, occipital lobe, and subcortical regions such as the thalamus, caudate, putamen, and globus pallidus (see Figure 2 and Table 2). 


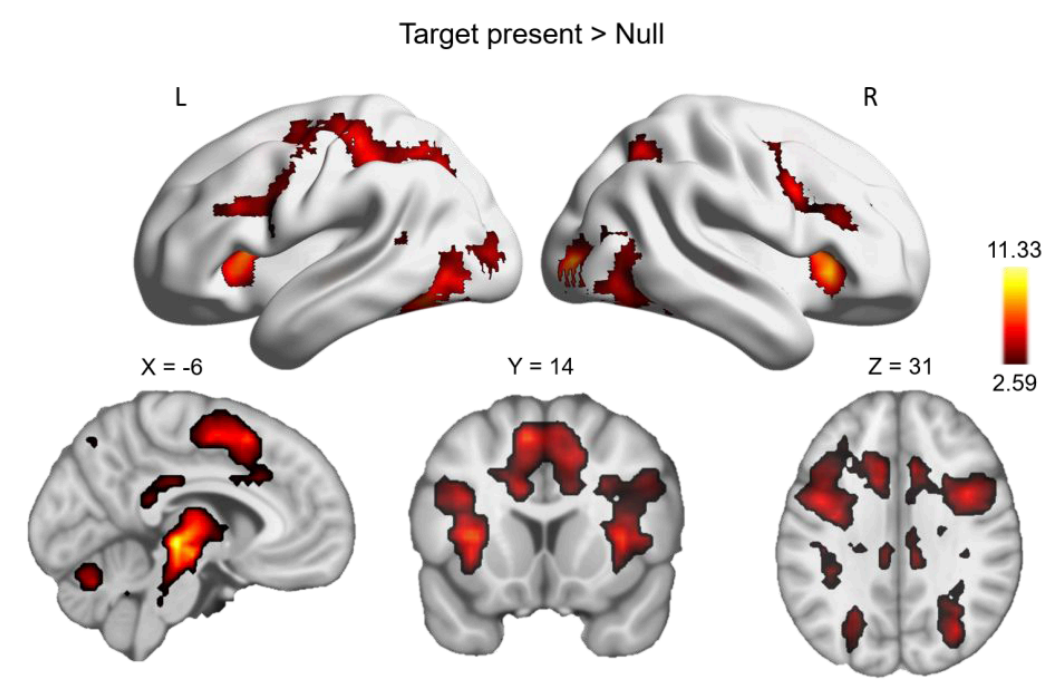

Figure 2. Brain renderings and sections showing activations for Target present $>$ Null whole-brain contrast ( $q \leq 0.05_{1}$ voxel-wise FDR corrected). The color bar denotes $t$-values. 
Table 2. Brain activations obtained in the whole-brain contrast Target present $>$ Null.

\begin{tabular}{|c|c|c|c|c|c|}
\hline Brain area & \multicolumn{3}{|c|}{ MNI $(x, y, z)$} & z-score & Voxels \\
\hline \multicolumn{6}{|l|}{ Frontal } \\
\hline L Insula & -33 & 17 & 7 & 5.11 & 165 \\
\hline R Insula & 33 & 23 & -2 & 5.56 & 117 \\
\hline L SMA & -6 & 14 & 52 & 4.81 & 262 \\
\hline R SMA & 6 & 11 & 55 & 4.65 & 152 \\
\hline L IFG & -33 & 17 & 10 & 4.78 & 180 \\
\hline R IFG & 36 & 26 & 7 & 4.98 & 162 \\
\hline L FEF & -24 & 10 & 49 & 4.76 & 21 \\
\hline R FEF & 36 & -1 & 52 & 4.17 & 21 \\
\hline L MFG & -30 & 38 & 16 & 3.66 & 74 \\
\hline L ACC & -9 & 23 & 28 & 3.49 & 6 \\
\hline R ACC & 9 & 20 & 28 & 3.40 & 23 \\
\hline \multicolumn{6}{|l|}{ Parietal } \\
\hline L IPL & -30 & -49 & 46 & 5.08 & 232 \\
\hline R IPL & 30 & -55 & 49 & 4.37 & 47 \\
\hline L SPL & -27 & -58 & 49 & 4.84 & 157 \\
\hline R SPL & 27 & -58 & 49 & 4.17 & 57 \\
\hline \multicolumn{6}{|l|}{ Occipital } \\
\hline L IO & -39 & -58 & -11 & 5.78 & 174 \\
\hline R IO & 39 & -64 & -11 & 6.13 & 154 \\
\hline L MO & -39 & -79 & -2 & 5.79 & 431 \\
\hline R MO & 36 & -88 & 4 & 5.36 & 267 \\
\hline L SO & -21 & -67 & 37 & 3.70 & 32 \\
\hline R So & 27 & -76 & 22 & 4.07 & 60 \\
\hline \multicolumn{6}{|l|}{ Subcortical } \\
\hline L Thalamus & -6 & 25 & -2 & 5.67 & 195 \\
\hline R Thalamus & 9 & -10 & 4 & 4.68 & 111 \\
\hline L Globus pallidus & -15 & -1 & -2 & 4.98 & 19 \\
\hline R Globus pallidus & 15 & 2 & -2 & 3.80 & 11 \\
\hline
\end{tabular}

ACC, anterior cingulate cortex; FEF, frontal eye field; IFG, inferior frontal gyrus; IO, inferior occipital; IPL, inferior parietal lobe; L, left; MFG, middle frontal gyrus; MO, middle occipital; R, right; SMA, supplementary motor area; SO, superior occipital; SPL, superior parietal lobe.

Parameter estimate ROI analyses were conducted for those areas previously related to conscious perception or executive control (see Methods section; Dehaene \& Changeux, 2011; Dehaene, Changeux, Naccache, Sackur, \& Sergent, 2006; Dosenbach et al., 2008). The analysis revealed a group of regions showing a statistically significant main effect of Congruency, with higher activations for incongruent than congruent trials. These regions included the bilateral IFG, left MFG, bilateral SMA, left FEF, left IPL, bilateral 
SPL, and (marginally) the left ACC. Another set of regions showed a statistically significant main effect of Awareness, with greater activations for seen than unseen trials. These regions included the left SMA, the bilateral FEF, the bilateral insula, the bilateral IPL, and the bilateral IPL (see Table 3). The right ACC and the right MFG were also analyzed and did not show any significant effect (all other $p s \geq 0.154$ ). No interaction between Congruency and Awareness was found in any of the regions here examined (all ps $\geq$ 0.153).

Table 3. ROls showing significant main effects of awareness and congruency in the parameter estimate analysis.

\begin{tabular}{|c|c|c|c|c|}
\hline Brain area & $\mathbf{F}$ & MSE & $\mathbf{p}$ & $\eta_{p}^{2}$ \\
\hline \multicolumn{5}{|c|}{ Main effect of Congruency } \\
\hline L IFG & 44.24 & 2.14 & $>0.001$ & 0.70 \\
\hline R IFG & 5.87 & 2.15 & 0.026 & 0.25 \\
\hline L IPL & 16.66 & 3.55 & 0.001 & 0.47 \\
\hline L SPL & 15.10 & 3.90 & 0.001 & 0.44 \\
\hline R SPL & 12.05 & 3.15 & 0.003 & 0.40 \\
\hline L SMA & 13.16 & 3.09 & 0.002 & 0.42 \\
\hline R SMA & 9.87 & 2.70 & 0.005 & 0.34 \\
\hline L MFG & 7.85 & 2.46 & 0.011 & 0.29 \\
\hline L FEF & 7.43 & 2.11 & 0.013 & 0.28 \\
\hline L ACC & 3.86 & 8.84 & 0.064 & 0.17 \\
\hline \multicolumn{5}{|c|}{ Main effect of Awareness } \\
\hline L FEF & 52.10 & 5.21 & $>0.001$ & 0.73 \\
\hline R FEF & 10.89 & 9.47 & 0.004 & 0.38 \\
\hline L IPL & 32.17 & 3.86 & $>0.001$ & 0.63 \\
\hline R IPL & 8.98 & 9.91 & 0.008 & 0.33 \\
\hline L SPL & 29.86 & 3.35 & $>0.001$ & 0.61 \\
\hline R SPL & 13.29 & 6.43 & 0.002 & 0.43 \\
\hline L Insula & 6.95 & 10.32 & 0.016 & 0.27 \\
\hline R Insula & 4.81 & 12.04 & 0.041 & 0.20 \\
\hline L SMA & 5.48 & 7.86 & 0.031 & 0.23 \\
\hline
\end{tabular}

ACC, anterior cingulate cortex; FEF, frontal eye field; IFG, inferior frontal gyrus; IPL, inferior parietal lobe; L, left; MFG, middle frontal gyrus; R, right; SMA, supplementary motor area; $\mathrm{SPL}$, superior parietal lobe. 
We then conducted pairwise functional connectivity analyses including the regions showing the Congruency effect in the previous ROls analyses. To explore if these areas showed significant coupling strength within each of the main four conditions of interest (i.e., congruent seen, congruent unseen, incongruent seen, incongruent unseen) pairwise beta-series correlation values were averaged per condition and two-tailed tests were used to determine the statistical significance of these $r$ values, correcting for multiple comparisons $(q<0.05$, FDR). This analysis revealed a strong frontal connectivity together with frontoparietal connectivity during the congruent seen condition. However, in the other three conditions, the connectivity within frontal regions seemed to be reduced and the frontoparietal connectivity was scarce or non-existent. Figure 3 shows the pairs of nodes that revealed significant functional coupling per each of the main conditions in our fMRI experimental design. 


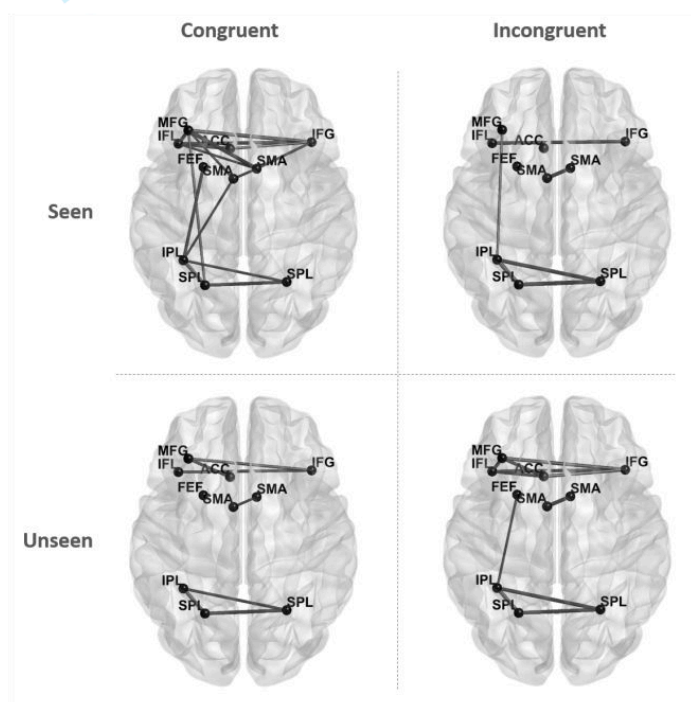

Figure 3. Pairwise functional connectivity between ROls showing the congruency main effect for each condition of interest. Edges denotes significant coupling ( $r$-values) among $\underline{\mathrm{ROls}}$ ( $\mathrm{q}<0.05$, FDR corrected). ACC, anterior cingulate cortex; FEF, frontal eye field; IFG, inferior frontal gyrus; IPL, inferior parietal lobe; MFG, middle frontal gyrus; SMA, supplementary motor area; SPL, superior parietal lobe.

Then, to specifically examine interactions between Congruency and Awareness in pairwise functional connectivity, we conducted repeatedmeasures ANOVAs for these pairs of regions using normalized mean Ztransformed values (see Methods section). Three pairs of regions showed a significant interaction between Congruency and Awareness in their functional connectivity: the left IFG and the left ACC, $F=8.50, M S E=1.64, p=0.011$, $\eta_{p}^{2}=0.38$, the left FEF and the left IPL, $F=7.67$, MSE $=1.55, p=0.014, \eta_{p}^{2}=$ 0.32 , and the right SMA and the right SPL, $F=5.03, \mathrm{MSE}=2.56, p=0.041$, $\eta_{p}^{2}=0.25$. In the congruent condition, these three pairs of regions showed stronger functional connectivity for seen compared to unseen trials. However, in the incongruent condition, the pairwise functional connectivity was not 
significantly different in any of the three pairs of regions for seen as compared to unseen trials (all ps $\geq 0.121$ ) (see Figure 4).
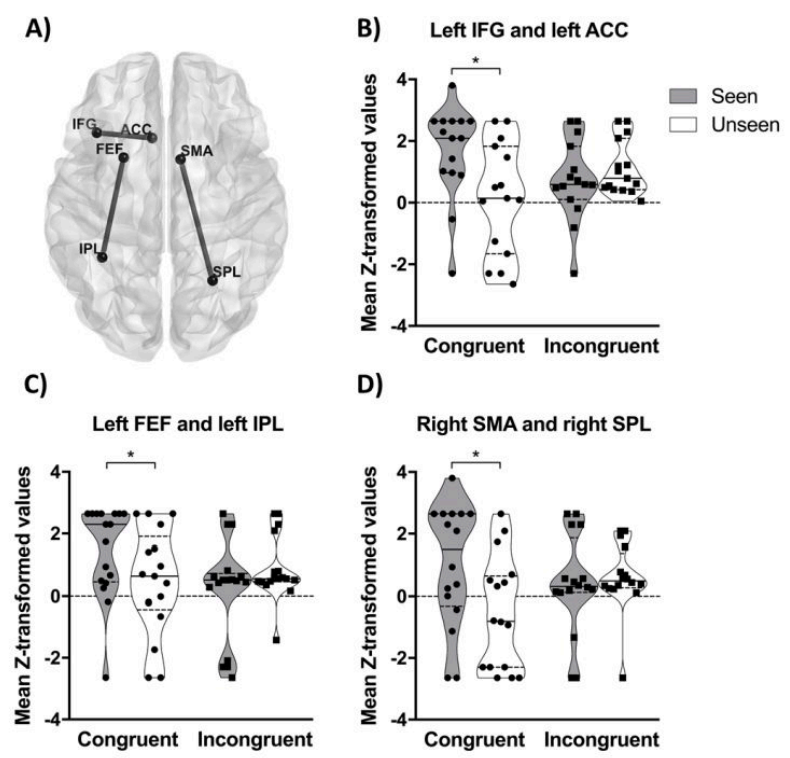

D)

Right SMA and right SPL

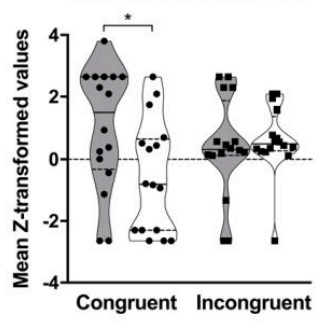

Figure 4. Graphical representation of the three pairs of regions demonstrating a significant interaction between Congruency and Awareness in their pairwise functional connectivity (A). Violin plots of the distribution of the mean Z-transformed values for the congruent $(\bullet)$ and incongruent $(\boldsymbol{\square})$ conditions and seen and unseen trials. The plots show the median (indicated by the solid horizontal lines) and quartiles (indicated by the dashed horizontal lines). Asterisks represent statistically significant T-tests (B, C, D).

Finally, due to the critical role of the ACC in executive control, we conducted whole-brain functional connectivity analysis using a seed placed in the left ACC region for each of the four conditions of interest in our experimental design. As it can be observed in Figure 5, during the unseen conditions, there was a significant functional coupling for the left ACC mainly with midline and lateral frontal regions. This connectivity was especially observed in the incongruent unseen condition. However, the functional coupling of the left ACC for the seen conditions was scarce or inexistent. 


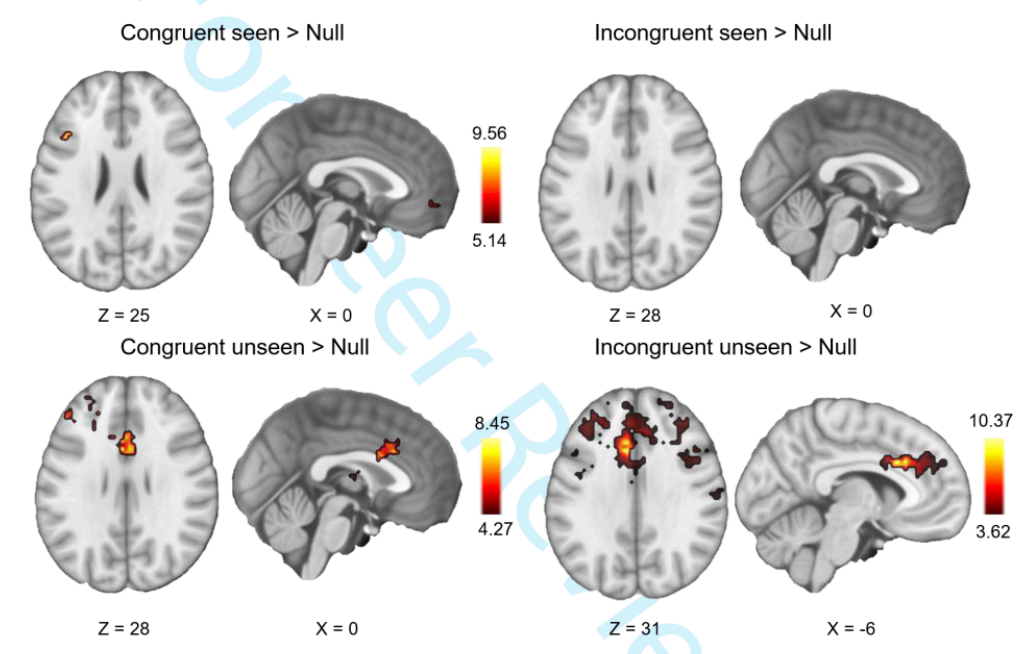

Figure 5. Brain regions showing whole-brain functional connectivity using the left $A C C$ as a seed for the four conditions of interest vs Null ( $q<0.05_{1}$ voxel-wise FDR corrected). The color bar denotes t-values.

\section{DWI tractography results}

We found a significant positive correlation between the HMOA of the left SLF II and the congruency effect over the functional connectivity between the left IFG-left ACC, $r=0.676, p=0.008, B_{10}=8.08$, and the right SMAright SPL, $r=0.655, p=0.008, \mathrm{BF}_{10}=7.83$. Higher HMOA of the left SLF II was associated with a larger congruency effect over the functional connectivity of these two pairs of frontoparietal regions (Figure 6). Additionally, we found a significant positive correlation between the HMOA of the left SLF I and the congruency effect over the percentage of seen targets, $r$ $=0.465, \mathrm{p}=0.045, \mathrm{BF}_{10}=1.86 . \mathrm{BF}$ analyses revealed that while the evidence in favor of the alternative hypothesis for the correlation between the behavioral congruency effect and the left SLF I HMOA resulted to be anecdotal, the evidence for the correlations between the pairwise functional 
connectivity and the left SLF II HMOA was substantial (Jarosz and Wiley 2014).
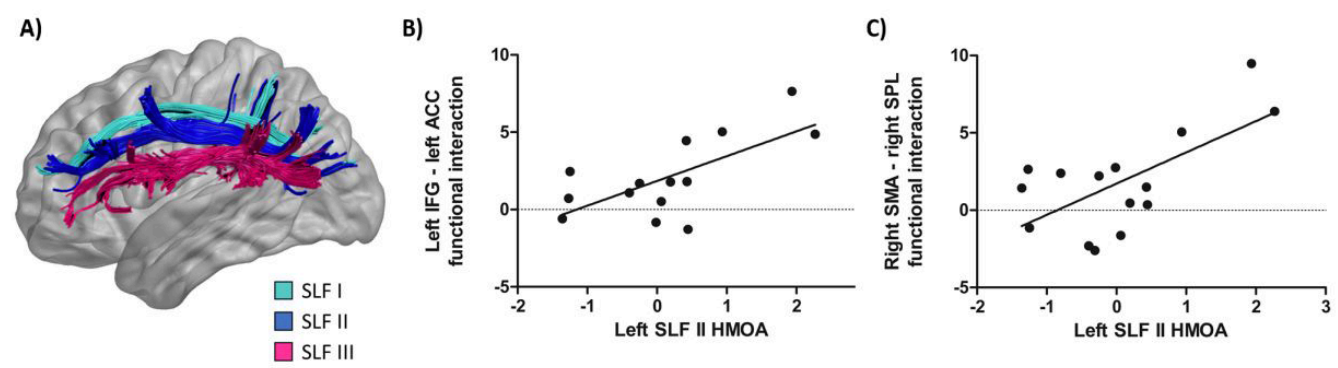

Figure 6. Example of a virtual in vivo dissection of the SLF I (light blue), II (dark blue), and III (pink) of the left hemisphere using deterministic tractography (A). Pearson correlations between the mean HMOA of the left SLF II and the interaction in functional connectivity of the left IFG and the left ACC (B), and the right SMA and the right SPL (C). Normalized data by Z score transformation. The black line represents the best fit of the data.

\section{DISCUSSION}

Conscious perception is impaired under situations of high conflict requiring activation of the executive control system (Meyer and Kieras 1997; Colás et al. 2017). The present study aimed at examining this interaction between executive control and consciousness at the neural level with a methodology allowing a good spatial resolution. To this end, we used an event-related fMRI design while manipulating executive control with a Stroop task in which congruent and incongruent stimuli were presented concurrently with near-threshold Gabor stimuli.

At the behavioral level, the Stroop task induced a reliable interference effect with longer RTs and lower accuracy for the incongruent compared to the congruent condition. Executive control also affected the conscious 
perception of the near-threshold Gabor, as fewer targets were perceived for the incongruent than the congruent condition, especially for those participants demonstrating larger RT effects on the Stroop task.

In dual-task paradigms, the conscious access of the second of two targets can be delayed in time (a phenomenon called the Psychological Refractory Period, Pashler, 1994) or it can even fail (like in the Attentional Blink phenomenon, Shapiro et al., 1997). According to the delayed conscious perception hypothesis, response selection of one task can block the conscious awareness of another stimulus presented concurrently or within a short interval. This is hypothesized to occur because conscious access and response selection are serial processes which cannot occur in parallel (Pashler 1994; Sigman and Dehaene 2008; Marti et al. 2012). In our paradigm, we did not analyze RTs to the conscious detection task as the response was given by the participants without time pressure. However, as in the Attentional Blink phenomenon, the conscious access of the Gabor stimulus may have been impaired in the incongruent Stroop trials as conflict detection and resolution on these trials require more time before selecting the response.

The present fMRI results showed that a group of frontal and parietal regions demonstrated larger BOLD responses for the incongruent than the congruent condition. Another group of frontoparietal regions demonstrated larger BOLD responses for seen than unseen Gabors. These results are in agreement with previous literature on cognitive control (Cole and Schneider 2007; Cocchi et al. 2013), and with some influential models proposing the 
importance of a distributed frontoparietal network for conscious perception (Dehaene and Naccache 2001; Dehaene et al. 2006).

Nevertheless, we did not find an interaction between executive control and consciousness in the ROI analysis for any of the frontoparietal regions here examined. Previous research manipulating spatial orienting and alerting while measuring conscious perception of near-threshold stimuli have demonstrated reliable interactions in frontoparietal (for spatial orienting, Chica, Paz-Alonso, Valero-Cabre, \& Bartolomeo, 2013) and frontostriatal (for alerting, Chica, Bayle, Botta, Bartolomeo, \& Paz-Alonso, 2016) regions. If the results were focused exclusively on ROI analyses, one could have concluded that executive attention and conscious perception exert their effects through independent brain networks. This result would have supported the cumulative influence hypothesis proposed by Tallon-Baudry (2012), according to which attention and conscious perception independently feed a decision-making mechanism implemented in the frontal lobe. This hypothesis predicts that attention and consciousness rely on distinct neural mechanisms, and therefore both processes should not interact at the neural level. Consistently, previous results have demonstrated that at least some attentional subsystems, such as endogenous attentional orienting, can be dissociated at the neural level from conscious perception (Wyart and Tallon-Baudry 2008; Chica et al. 2012; Tallon-Baudry 2012).

However, after exploring functional connectivity, we did find an interaction between executive control and consciousness in the functional coupling of three frontoparietal pairs of regions: left IFG-left ACC, left FEF-left IPL, and right SMA-right SPL. Importantly, these pairs of regions were more 
strongly coactivated when the Gabor was consciously seen than when it was not consciously seen for congruent Stroop trials, while the coactivation of these regions was similar for seen and unseen targets on incongruent Stroop trials. One interpretation of these results is that frontoparietal connectivity is important for consciousness in situations of low conflict (i.e., congruent conditions), but when conflict is experienced (i.e., incongruent conditions), frontoparietal connectivity is not associated with conscious perception. An alternative possibility is that this frontoparietal connectivity is important for the conscious perception of near-threshold targets (as shown in Chica et al. 2013). Thus, in conditions where conflict is experienced (i.e., incongruent trials), parts of these networks could be recruited to solve the conflict, and be thus unavailable to sustain conscious perception, hence the absence of normal frontoparietal correlation ${ }^{1}$.

Our data are consistent with models such as the Gateway Hypothesis or the Global Neural Workspace, which postulate that attention modulates conscious perception either by increasing perceptual gain or biasing decision mechanisms (Reynolds and Chelazzi 2004; De Lange et al. 2011). The manipulation of executive control with the Stroop task involves conflict detection and resolution, cognitive processes in which frontal lobe structures such as the ACC and the dIPFC are crucial (Pardo et al. 1990; Macdonald et al. 2000; Milham et al. 2001; Egner and Hirsch 2005; Nee et al. 2007). This system would share neural resources with conscious perception, and therefore an interaction between executive attention and conscious perception was expected in frontal regions.

\footnotetext{
${ }^{1}$ We would like to thank an anonymous reviewer for this suggestion.
} 
Due to the critical role of the ACC for conflict evaluation and monitoring, we also explored functional connectivity with a seed placed in this region. We found a functional coupling between the left ACC and other frontal regions for the incongruent unseen condition, which was reduced or inexistent in the other three conditions. This enhanced functional connectivity between the left ACC and frontal regions was observed in the condition with a higher amount of conflict: incongruent Stroop trials in which the Gabor was missed.

Neuroimaging studies examining divided attention and the attentional blink phenomenon have linked these processes with the functioning of a frontoparietal network, with a critical role of left frontal areas (Fagioli and Macaluso 2009; Salo et al. 2017; Yaple and Vakhrushev 2018). Damage in the frontal lobes is also associated with impairments in temporal selection of visual stimuli and divided attention (Godefroy and Rousseaux 1996; Correani and Humphreys 2011). Studies in dual-tasks examining the neural mechanism of the Psychological Refractory Period point out to a large parietofrontal network, with a critical role of the lateral prefrontal cortex (Szameitat et al. 2002; Schubert and Szameitat 2003; Sigman and Dehaene 2008; Strobach et al. 2018). Therefore, being able to concurrently solve the Stroop task and to consciously detect the appearance of the Gabor may require the activity of frontal areas and their connection with parietal regions.

A further aim of this work was to explore the role of white matter fibers connecting the parietal and the frontal lobes in the interaction between executive attention and conscious perception. Recent work has linked the microstructure of the ventral branch of the SLF with diverse behavioral and neural measures related to the interaction between alerting and orienting 
attentional mechanisms with conscious perception. The microstructure of the ventral branch of the left SLF predicted the neural interactions (measured with $\mathrm{fMRI}$ ) observed between alerting and orienting attentional mechanisms and conscious perception (Chica et al. 2018). Moreover, the microstructure of the ventral branch of the right SLF also modulated the effect caused by transcranial magnetic stimulation (TMS) over the SMA in a conscious perception task preceded by an alerting signal. In this case, the more increased the HMOA of the right SLF III, the more reduced the TMS effects (Martín-Signes et al. 2017). In this study, we correlated behavioral and neural effects of the executive attentional modulations over conscious perception with the microstructure of the dorsal, middle, and ventral branches of the SLF. We found a positive correlation between the HMOA of the left SLF II and the functional connectivity measures of the interaction between executive control and perceptual consciousness.

Although research linking white matter microstructure and behavioral or functional data is still scarce, this investigation contributes to the knowledge of the functional role of the different branches of the SLF in attentional mechanisms (Parlatini et al. 2017). While the ventral branch seems to be involved in bottom-up processes (such us alerting and orienting), the more dorsal branches (i.e. SLF II and maybe SLF I) seem to be involved in topdown processes (such as executive control). Regarding patients, one study has linked the white matter volume of the SLF with impairments in executive control measures (Blanc et al. 2012) while another study found structural white matter abnormalities, including the SLF, in attention-deficit/hyperactivity disorder (Makris et al. 2008); however, these studies did not distinguish 
between the different branches of the SLF. To our knowledge, the present study is the first one examining the role of the SLF in executive control in healthy population. However, we reckon that due to the sample size of the study, this correlational analysis should be considered merely exploratory, and conclusions drawn with caution.

To conclude, our data support the gateway theory about the relationship between attention and consciousness (Posner 1994; Dehaene and Naccache 2001; Dehaene et al. 2006). Executive control modulated the conscious perception of near-threshold stimuli, which, at the neural level, was reflected in the functional connectivity of frontoparietal regions. DWI analysis highlighted the role of the middle branch of the SLF in the interaction between executive control and consciousness. This study demonstrates the importance of taking into account functional and structural connectivity measures for a more complete understanding of the neural mechanisms supporting executive attention and consciousness interactions. 


\section{REFERENCES}

Abdi H. 2007. Signal Detection Theory ( SDT ). Encycl Meas Stat. 1-9.

Blanc F, Noblet V, Jung B, Rousseau F, Renard F, Bourre B, Longato N, Cremel N, Di Bitonto L, Kleitz C, Collongues N, Foucher J, Kremer S, Armspach JP, de Seze J. 2012. White matter atrophy and cognitive dysfunctions in neuromyelitis optica. PLoS One. 7.

Botta F, Lupiáñez J, Chica AB. 2014. When endogenous spatial attention improves conscious perception: Effects of alerting and bottom-up activation. Conscious Cogn. 23:63-73.

Botvinick MM, Braver TS, Barch DM, Carter CS, Cohen JD. 2001. Conflict monitoring and cognitive control. Psychol Rev. 108:624-652.

Bourgeois A, Chica AB, Migliaccio R, Bayle DJ, Duret C, Pradat-Diehl P, Lunven M, Pouget P, Bartolomeo P. 2015. Inappropriate rightward saccades after right hemisphere damage: Oculomotor analysis and anatomical correlates. Neuropsychologia. 73:1-11.

Bressler SL, Menon V. 2010. Large-scale brain networks in cognition: emerging methods and principles. Trends Cogn Sci. 14:277-290.

Brett M, Anton J-LL, Valabregue R, Poline J-B. 2002. Region of interest analysis using an SPM toolbox - Abstract Presented at the 8th International Conference on Functional Mapping of the Human Brain, June 2-6, 2002, Sendai, Japan. Neuroimage. 16:Abstract 497.

Carretié L, Ríos M, Periáñez JA, Kessel D, Álvarez-Linera J. 2012. The role of low and high spatial frequencies in exogenous attention to biologically 
salient stimuli. PLoS One. 7:1-8.

Chiang H-L, Chen Y-J, Lo Y-C, Tseng W-YI, Gau SS. 2015. Altered white matter tract property related to impaired focused attention, sustained attention, cognitive impulsivity and vigilance in attention-deficit/ hyperactivity disorder. J Psychiatry Neurosci. 40:325-335.

Chica AB, Bartolomeo P. 2012. Attentional routes to conscious perception. Front Psychol. 3:1-12.

Chica AB, Bayle DJ, Botta F, Bartolomeo P, Paz-Alonso PM. 2016. Interactions between phasic alerting and consciousness in the frontostriatal network. Sci Rep. 6:31868.

Chica AB, Botta F, Lupiáñez J, Bartolomeo P. 2012. Spatial attention and conscious perception: interactions and dissociations between and within endogenous and exogenous processes. Neuropsychologia. 50:621-629.

Chica AB, Lasaponara S, Lupiáñez J, Doricchi F, Bartolomeo P. 2010. Exogenous attention can capture perceptual consciousness: ERP and behavioural evidence. Neuroimage. 51:1205-1212.

Chica AB, Paz-Alonso PM, Valero-Cabre A, Bartolomeo P. 2013. Neural Bases of the Interactions between Spatial Attention and Conscious Perception. Cereb Cortex. 23:1269-1279.

Chica AB, Thiebaut de Schotten M, Bartolomeo P, Paz-Alonso PM. 2018. White matter microstructure of attentional networks predicts attention and consciousness functional interactions. Brain Struct Funct. 653-668.

Ciaraffa F, Castelli G, Parati EA, Bartolomeo P, Bizzi A. 2013. Visual neglect 
as a disconnection syndrome? A confirmatory case report. Neurocase. 19:351-359.

Cocchi L, Zalesky A, Fornito A, Mattingley JB. 2013. Dynamic cooperation and competition between brain systems during cognitive control. Trends Cogn Sci. 17:493-501.

Cocosco CA, Kollokian V, Kwan RK, Pike GB, Evans AC. 1997. BrainWeb: Online Interface to a 3D MRI Simulated Brain Database. 3-rd Int Conf Funct Mapp Hum Brain. 5:S425.

Colás I, Capilla A, Chica AB. 2018. Neural modulations of interference control over conscious perception Itsaso. Neuropsychologia. 112:40-49.

Colás I, Triviño M, Chica AB. 2017. Interference control modulations over conscious perception. Front Psychol. 8:1-12.

Cole MW, Schneider W. 2007. The cognitive control network: Integrated cortical regions with dissociable functions. Neuroimage. 37:343-360.

Correani A, Humphreys GW. 2011. An impaired attentional dwell time after parietal and frontal lesions related to impaired selective attention not unilateral neglect. Cogn Neuropsychol. 28:363-385.

Dale AM. 1999. Optimal experimental design for event-related fMRI. Hum Brain Mapp. 8:109-114.

De Lange FP, Van Gaal S, Lamme VAF, Dehaene S. 2011. How awareness changes the relative weights of evidence during human decision-making. PLoS Biol. 9:1-10.

Dehaene S, Changeux JP. 2011. Experimental and theoretical approaches to 
conscious processing. Neuron. 70:200-227.

Dehaene S, Changeux JP, Naccache L, Sackur J, Sergent C. 2006. Conscious, preconscious, and subliminal processing: a testable taxonomy. Trends Cogn Sci. 10:204-211.

Dehaene S, Naccache L. 2001. Towards a cognitive neuroscience of consciousness: Basic evidence and a workspace framework. Cognition. 79:1-37.

Dell'Acqua F, Simmons A, Williams SCR, Catani M. 2013. Can spherical deconvolution provide more information than fiber orientations? Hindrance modulated orientational anisotropy, a true-tract specific index to characterize white matter diffusion. Hum Brain Mapp. 34:2464-2483.

Diamond A. 2013. Executive Functions. Annu Rev Psychol. 64:135-168.

Dienes Z, Mclatchie N. 2018. Four reasons to prefer Bayesian analyses over significance testing. Psychon Bull Rev. 25:207-218.

Doricchi F, Thiebaut de Schotten M, Tomaiuolo F, Bartolomeo P. 2008. White matter (dis)connections and gray matter (dys)functions in visual neglect: Gaining insights into the brain networks of spatial awareness. Cortex. 44:983-995.

Dosenbach NUF, Fair DA, Cohen AL, Schlaggar BL, Petersen SE. 2008. A dual-networks architecture of top-down control. Trends Cogn Sci. 12:99105.

Egner T, Hirsch J. 2005. The neural correlates and functional integration of cognitive control in a Stroop task. Neuroimage. 24:539-547. 
Fagioli S, Macaluso E. 2009. Attending to multiple visual streams: interactions between location-based and category-based attentional selection. J Cogn Neurosci. 21:1628-1641.

Fan J, McCandliss BD, Fossella J, Flombaum JI, Posner MI. 2005. The activation of attentional networks. Neuroimage. 26:471-479.

Fisher RA. 1921. On the mathematical foundations of theoretical statistics. Philos Trans R Soc A Math Phys Eng Sci. 222:309-368.

Godefroy O, Rousseaux M. 1996. Divided and focused attention in patients with lesion of the Prefrontal Cortex. Brain Cogn. 30:155-174.

Jarosz AF, Wiley J. 2014. What are the odds? A practical guide to computing and reporting Bayes Factors. J Probl Solving. 7:2-9.

Klarborg B, Skak Madsen K, Vestergaard M, Skimminge A, Jernigan TL, Baaré WFC. 2013. Sustained attention is associated with right superior longitudinal fasciculus and superior parietal white matter microstructure in children. Hum Brain Mapp. 34:3216-3232.

Koch C, Tsuchiya N. 2007. Attention and consciousness: two distinct brain processes. Trends Cogn Sci. 11:16-22.

Konrad A, Dielentheis TF, El Masri D, Bayerl M, Fehr C, Gesierich T, Vucurevic G, Stoeter P, Winterer G. 2010. Disturbed structural connectivity is related to inattention and impulsivity in adult attention deficit hyperactivity disorder. Eur J Neurosci. 31:912-919.

Kusnir F, Chica AB, Mitsumasu M a., Bartolomeo P. 2011. Phasic auditory alerting improves visual conscious perception. Conscious Cogn. 
20:1201-1210.

Lau H, Rosenthal D. 2011. Empirical support for higher-order theories of conscious awareness. Trends Cogn Sci. 15:365-373.

Leemans A, Jeurissen B, Sijbers J, Jones DK. 2009. ExploreDTI: a graphical toolbox for processing, analyzing, and visualizing diffusion MR data. $17: 3537$.

Macdonald AW, Cohen JD, Stenger VA, Carter CS. 2000. Dissociating the role of the Dorsolateral Prefrontal and Anterior Cingulate Cortex in cognitive control. Science. 288:1835-1838.

Makris N, Buka SL, Biederman J, Papadimitriou GM, Hodge SM, Valera EM, Brown AB, Bush G, Monuteaux MC, Caviness VS, Kennedy DN, Seidman LJ. 2008. Attention and executive systems abnormalities in adults with childhood ADHD: a DT-MRI study of connections. Cereb Cortex May. 18:1210-1220.

Marti S, Sigman M, Dehaene S. 2012. A shared cortical bottleneck underlying attentional blink and psychological refractory period. Neuroimage. 59:2883-2898.

Martín-Signes M, Pérez-Serrano C, Chica AB. 2017. Causal contributions of the SMA to alertness and consciousness interactions. Cereb Cortex. 1-9.

Meyer DE, Kieras DE. 1997. A computational theory of executive control processes and human multiple-task performance: Part 1. Basic Mechanisms. Psychol Rev. 104:3-65.

Milham MP, Banich MT, Webb A, Barad V, Cohen NJ, Wszalek T, Kramer AF. 
2001. The relative involvement of anterior cingulate and prefrontal cortex in attentional control depends on nature of conflict. Cogn Brain Res. $12: 467-473$.

Miller EK, Cohen JD. 2001. An integrative theory of Prefrontal Cortex function. Annu Rev Neurosci. 24:167-202.

Nee DE, Wager TD, Jonides J. 2007. Interference resolution: insights from a meta-analysis of neuroimaging tasks. Cogn Affect Behav Neurosci. 7:117.

Norman DA, Shallice T. 1986. Attention to attention to action: willed and automatic control of behaviour. In: Consciousness and Self-Regulation. Boston, MA: Springer US. p. 1-18.

Pardo J V, Pardo PJ, Janer KW, Raichle ME. 1990. The anterior cingulate cortex mediates processing selection in the Stroop attentional conflict paradigm. Proc Natl Acad Sci. 87:256-259.

Parlatini V, Radua J, Dell'Acqua F, Leslie A, Simmons A, Murphy DG, Catani M, Thiebaut de Schotten M. 2017. Functional segregation and integration within fronto-parietal networks. Neuroimage. 146:367-375.

Pashler H. 1994. Dual-task interference in simple tasks: Data and theory. Psychol Bull. 116:220-244.

Petersen A, Petersen AH, Bundesen C, Vangkilde S, Habekost T. 2017. The effect of phasic auditory alerting on visual perception. Cognition. 165:7381.

Petersen, Posner M. 2012. The attention system of the human brain: 20 years 
after. Annu Rev Neurosci. 21:73-89.

Posner MI. 1994. Attention: the mechanisms of consciousness. Proc Natl Acad Sci U S A. 91:7398-7403.

Posner MI, Digirolamo GJ. 1998. Executive attention: Conflict, target detection, and cognitive control. R Parasuraman.

Posner MI, Petersen SE. 1990. The attention system of the human brain. Annu Rev Neurosci. 13:25-42.

Rees G, Kreiman G, Koch C. 2002. Neural correlates of consciousness in humans. Nat Rev Neurosci. 3:261-270.

Reynolds JH, Chelazzi L. 2004. Attentional modulation of visual processing. Annu Rev Neurosci. 27:611-647.

Rissman J, Gazzaley A, D 'esposito M, Wheeler HH. 2004. Measuring functional connectivity during distinct stages of a cognitive task. Neuroimage. 23:752-763.

Rojkova K, Volle E, Urbanski M, Humbert F, Dell'Acqua F, Thiebaut de Schotten M. 2016. Atlasing the frontal lobe connections and their variability due to age and education: a spherical deconvolution tractography study. Brain Struct Funct. 221:1751-1766.

Rounis E, Maniscalco B, Rothwell JC, Passingham RE, Lau H. 2010. Thetaburst transcranial magnetic stimulation to the prefrontal cortex impairs metacognitive visual awareness. Cogn Neurosci. 1:165-175.

Salo E, Salmela V, Salmi J, Numminen J, Alho K. 2017. Brain activity associated with selective attention, divided attention and distraction. 
Brain Res. 1664:25-36.

Schneider W, Eschman A, Zuccolotto A, Burgess S, Cernicky B, Gilkey D, Gliptis J, Maciejczyk V, Macwhinney B, Rodgers K, James JS. 2002. EPrime user's guide. Pittsburgh: Psychology Software Tools, Inc.

Schubert T, Szameitat AJ. 2003. Functional neuroanatomy of interference in overlapping dual tasks: An fMRI study. Cogn Brain Res. 17:733-746.

Shapiro KL, Arnell KM, Raymond JE. 1997. The attentional blink. Trends Cogn Sci. 1:291-296.

Sigman M, Dehaene S. 2008. Brain mechanisms of serial and parallel processing during dual-task performance. J Neurosci. 28:7585-7598.

Simons DJ, Levin DT. 1997. Change blindness. Trends Cogn Sci. 1:261-267.

Solomon JA. 2004. The effect of spatial cues on visual sensitivity. Vision Res. $44: 1209-1216$.

Stanislaw H, Todorov N. 1999. Calculation of signal detection theory measures. Behav Res Methods, Instruments, Comput. 3:37-149.

Strobach T, Antonenko D, Abbarin M, Escher M, Flöel A, Schubert T. 2018. Modulation of dual-task control with right prefrontal transcranial direct current stimulation (tDCS). Exp Brain Res. 236:227-241.

Szameitat AJ, Schubert T, Müller K, Von Yves Cramon D. 2002. Localization of executive functions in dual-task performance with fMRI. J Cogn Neurosci. 14:1184-1199.

Tallon-Baudry C. 2012. On the neural mechanisms subserving consciousness 
and attention. Front Psychol. 3:1-11.

Thiebaut de Schotten M, Dell'Acqua F, Forkel SJ, Simmons A, Vergani F, Murphy DGM, Catani M. 2011. A lateralized brain network for visuospatial attention. Nat Neurosci. 14:1245-1247.

Thiebaut De Schotten M, Tomaiuolo F, Aiello M, Merola S, Silvetti M, Lecce F, Bartolomeo P, Doricchi F. 2014. Damage to white matter pathways in subacute and chronic spatial neglect: A group study and 2 single-case studies with complete virtual "in vivo" tractography dissection. Cereb Cortex. 24:691-706.

Vallar G, Bello L, Bricolo E, Castellano A, Casarotti A, Falini A, Riva M, Fava E, Papagno C. 2014. Cerebral correlates of visuospatial neglect: A direct cerebral stimulation study. Hum Brain Mapp. 35:1334-1350.

Wang R, Benner T, Sorensen AG, Wedeen VJ. 2007. Diffusion toolkit: A software package for diffusion imaging data processing and tractography. Proc Intl Soc Mag Reson Med. 15:3720.

Wolfers T, Onnink AMH, Zwiers MP, Arias-Vasquez A, Hoogman M, Mostert JC, Kan CC, Slaats-Willemse D, Buitelaar JK, Franke B. 2015. Lower white matter microstructure in the superior longitudinal fasciculus is associated with increased response time variability in adults with attention-deficit/ hyperactivity disorder. J Psychiatry Neurosci. 40:344351.

Wyart V, Tallon-Baudry C. 2008. Neural dissociation between visual awareness and spatial attention. J Neurosci. 28:2667-2679. 
Yaple Z, Vakhrushev R. 2018. Modulation of the frontal-parietal network by low intensity anti-phase $20 \mathrm{~Hz}$ transcranial electrical stimulation boosts performance in the attentional blink task. Int J Psychophysiol. 127:11-16. 